\title{
The brown midrib3 (bm3) Mutation in Maize Occurs in the Gene Encoding Caffeic Acid O-Methyltransferase
}

\author{
Florence Vignols, Joan Rigau, Miguel Angel Torres, Montserrat Capellades, and Pere Puigdomènech ${ }^{1}$ \\ Departament de Genètica Molecular, Centro de Investigación y Desarrollo, Consejo Superior de Investigaciones Científicas, \\ Jordi Girona 18, 08034 Barcelona, Spain
}

\begin{abstract}
The brown midrib mutations are among the earliest described in maize. Plants containing a brown midrib mutation exhibit a reddish brown pigmentation of the leaf midrib starting when there are four to six leaves. These mutations are known to alter lignin composition and digestibility of plants and therefore constitute prime candidates in the breeding of silage maize. Here, we show that two independent brown midrib3 (bm 3$)$ mutations have resulted from structural changes in the COMT gene, which encodes the enzyme O-methyltransferase (COMT; EC 2.1.1.6), involved in lignin biosynthesis. Our results indicate that the bm3-1 allele (the reference mutant allele) has arisen from an insertional event producing a COMT mRNA altered in both size and amount. By sequencing a COMT cDNA clone obtained from bm3-1 maize, a retrotransposon with homology to the B5 element has been found to be inserted near the junction of the $3^{\prime}$ coding region of the COMT gene intron. The second $b m 3$ allele, bm3-2, has resulted from a deletion of part of the COMT gene. These alterations of the COMT gene were confirmed by DNA gel blot and polymerase chain reaction amplification analyses. These results clearly demonstrate that mutations at the COMT gene give a brown midrib3 phenotype. Thus, the gene genetically recognized as $b m 3$ is the same as the one coding for COMT.
\end{abstract}

\section{INTRODUCTION}

Lignins are universal components in plants that form crosslinks with carbohydrates, such as hemicellulose in the cell wall (for review, see Lewis and Yamamoto, 1990). The resulting amorphous matrix provides the plant with multiple advantages, such as mechanical strength, resistance to pathogens, and waterproof qualities. However, due to their chemical properties, lignins are aiso responsible for the low agronomic and industrial value of a number of species. Because lignins are strongly linked to other cell wall constituents, they are difficult to extract or to degrade (Sarkanen and Ludwig, 1971). Thus, lignins appear to be an undesirable constituent that increases manufacturers' costs. Once extracted, lignin byproducts produce an adverse impact on the environment. Agronomically, lignin polymers have been shown to lower fiber digestion in ruminants, and it has been proposed that the degree of lignification is inversely proportional to forage crop digestibility (Grand et al., 1985; Cherney et al., 1991). Lignin biosynthesis has been studied extensively both because it is intrinsically interesting scientifically and because it can be used to decrease lignin content in plants of economic interest.

Lignins are biosynthesized through a complex pathway that involves various enzymatic activities and polymerization steps. The first enzymes required for lignin biosynthesis are those from the phenylpropanoid pathway, such as phenylalanine ammonia-lyase and cinnamate 4-hydroxylase (Lewis and

\footnotetext{
1 To whom correspondence should be addressed.
}

Yamamoto, 1990). Later, a series of hydroxylations and methylations involving various hydroxylases and an O-methyltransferase (OMT) provides cinnamic acid derivatives. These compounds are finally converted to respective alcohols by cinnamoylcoenzymeA reductase and cinnamyl alcohol dehydrogenase (CAD) (Lewis and Yamamoto, 1990). Different approaches designed to produce plants containing low lignin content include backcross selection, chemical mutagenesis (Cherney et al., 1991), and production of antisense plants for various enzymes involved in the lignin pathway (Dwivedi et al., 1993; Halpin et al., 1994; Ni et al., 1994). More logically, special attention has been devoted to plants carrying naturally occurring mutations and exhibiting this property.

The first brown midrib mutant was described in maize as early as 1924, in Minnesota (Jorgenson, 1931; Cherney et al., 1991). Plants possessing a brown midrib mutation exhibit a reddish brown pigmentation in the leaf midrib, which is initially visible when there are four to six leaves. As the plant grows and the stem lignifies, the reddish brown pigmentation is observed to be associated with vascular bundles and lignified rings in stem cross-sections. Later, the brown midrib phenotype gradually disappears from external exposed surfaces of the mature plant, but it is still detectable in internal and lignified tissues. Based on morphologic and genetic descriptions in maize, four mutations (brown midrib1 [bm1], bm2,bm3, and bm4) located on chromosomes 5, 1, 4, and 9, respectively (Neuffer et al., 1968), were shown to produce this phenotype. 
Additional analyses of these mutants indicated that the brown midrib mutations could be associated with lower quantity and different quality of lignins (Kuc et al., 1964, 1968; Gentinetta et al., 1990). Moreover, the mutants appeared to be more easily digested by ruminants (Barnes et al., 1971; Lechtenberg et al., 1972). These studies essentially dealt with $b m 3$ maize plants (and bm1 to a lesser extent) because the properties of this mutant (cell wall content and composition, agronomic values, and mechanical properties) were shown to be the most appropriate for breeding and feeding purposes (Barnes et al., 1971; Lechtenberg et al., 1972; Barrière and Argillier, 1993; Barrière et al., 1994). For these reasons, the brown midrib mutants, and especially $b m 3$, have been selected as a forage crop. However, the types of mutations that have occurred in brown midrib mutants and their relationship with the lignification process are still not known.

Our group recently characterized the first gene encoding a lignin-related OMT in maize (Collazo et al., 1992). We showed that this gene encodes a bifunctional enzyme able to methylate both caffeic and 5-hydroxyferulic acids to produce ferulic and sinapic acids, respectively. This caffeic acid OMT (COMT; EC 2.1.1.6) protein is encoded by a single gene, although another OMT-related gene ( $44 \%$ homologous to the previous one) has been described in maize and is probably involved in the suberin biosynthesis pathway (Held et al., 1993). The ligninrelated COMT gene is expressed in tissues undergoing lignification; these include elongating roots and vascular bundles in leaves (Collazo et al., 1992). A similar expression has been described for a number of other lignin-related OMT cDNAs (Bugos et al., 1991; Gowri et al., 1991; Dumas et al., 1992; Jaeck et al., 1992; Pellegrini et al., 1993). The obvious importance of maize brown midrib mutants and the availability of a COMT probe from maize allowed us to analyze further the effect of the brown midrib mutation on the expression of the COMT gene. The experiments described here report the molecular characterization of the lignification-related $b m 3$ mutation in maize.

\section{RESULTS}

\section{COMT Gene Expression Is Modified by the bm 3 Mutation}

The maize COMT gene is known to be strongly expressed in roots and to a lesser extent in the stem and in leaves (Collazo et al., 1992). To determine whether any of the brown midrib mutations modified COMT gene expression, different tissues of various brown midrib mutants were subjected to RNA gel blot analysis. RNA was first extracted from roots harvested from $b m 1, b m 2$, and $b m 3$ homozygote mutants maintained in W23 $\times$ L317 hybrids and hybridized with the $5^{\prime}$ coding region (XS1, Figure $1 \mathrm{~A}$ ) of the wild-type maize COMT gene as a probe. Figure $1 \mathrm{~B}$ shows that $b m 1$ and $b m 2$ mutants exhibited the same expression pattern, as did the wild type. Thus, neither $b m 1$ nor bm2 mutations appeared to alter COMT mRNA accumulation,

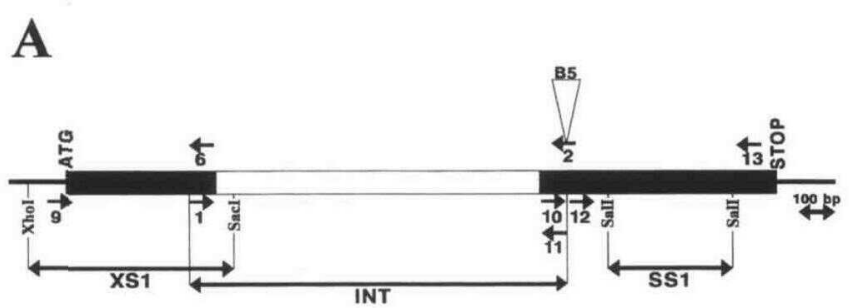

B
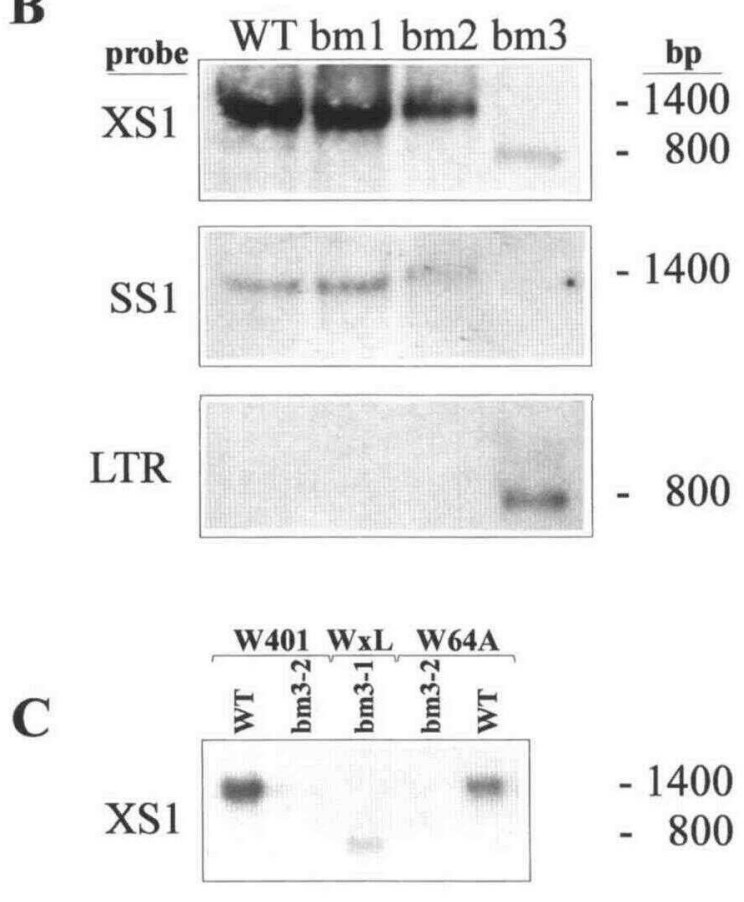

Figure 1. Expression of the Maize COMT Gene in Different brown midrib Mutants.

(A) Schematic representation of the MG18 COMT gene (Collazo et al., 1992). Exons are shown as solid boxes and the single intron as an open box. The positions of the different probes used in hybridization procedures are indicated below the scheme. Oligonucleotides used for PCR experiments are numbered and marked with arrows above and below the gene. The insertion site of the B5 element in the COMT is indicated with an open triangle.

(B) COMT mRNA accumulation in roots from brown-midrib mutants maintained in W23 $\times$ L317 hybrids. RNA gel blot analysis was performed from wild-type (WT) or brown-midrib homozygote plants for three different loci: brown-midrib1 (bm1), brown-midrib2 (bm2), and brownmidrib3 (bm3). Probes XS1 and SS1 shown in (A) or LTR were radiolabeled and used to hybridize to the blot. The length of the hybridizing bands is indicated at right in base pairs.

(C) COMT mRNA accumulation in roots from brown-midrib3 mutants maintained in pure lines. RNA gel blot analysis was performed from bm 3 homozygote plants maintained in W64A and W401 pure lines (bm3-2). WT refers to the W64A and W401 wild-type plants, and bm3-1 refers to the brown-midrib3 homozygote from (B) maintained in W23 $\times$ L317 hybrid $(\mathrm{W} \times \mathrm{L})$. The length of the hybridizing bands is indicated at right in base pairs. 


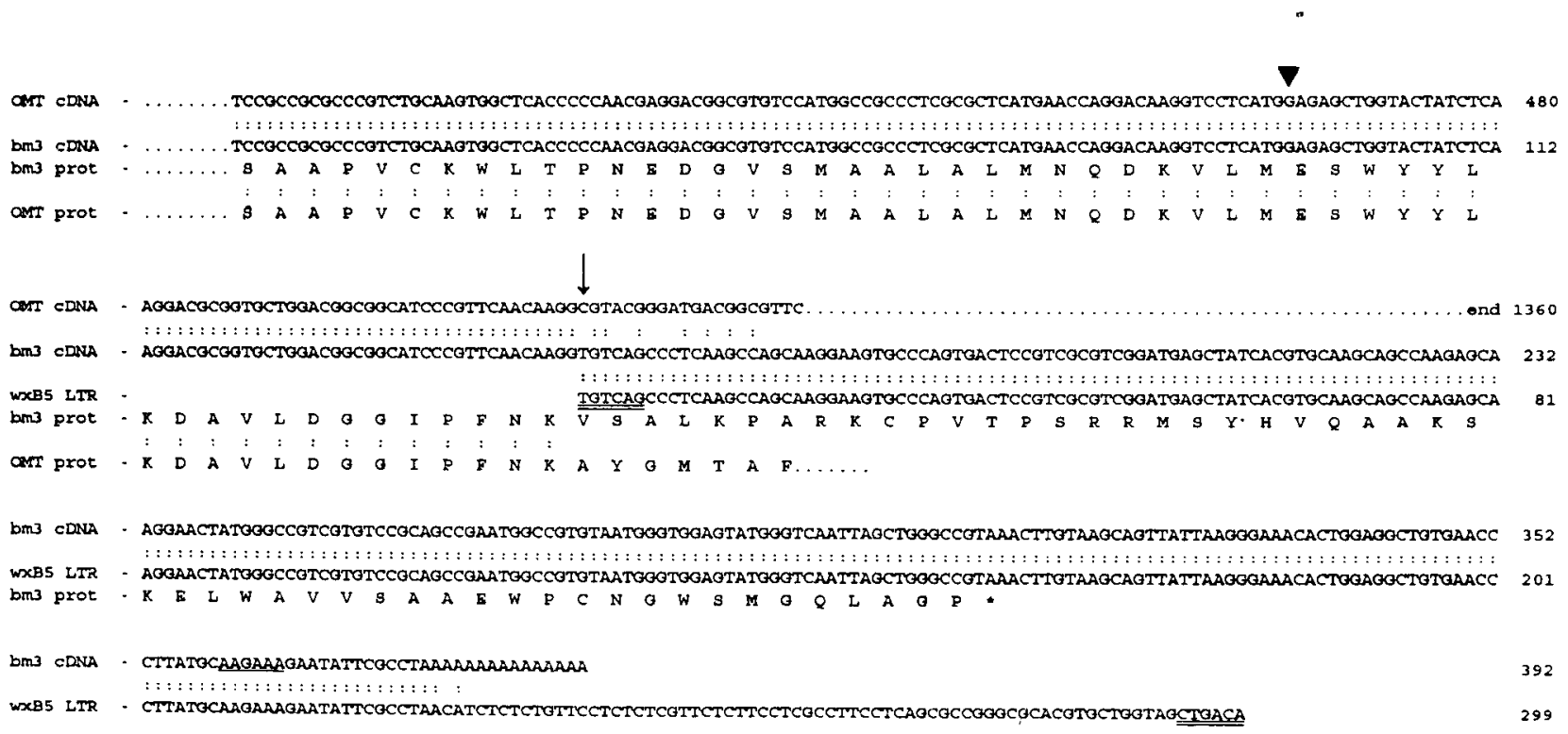

Figure 2. Comparison of COMT CDNA Sequences Obtained from Wild-Type W64A and the bm3-1 Mutant of Maize.

The sequence alignment involves COMT sequences of the wild-type MC1 (OMT cDNA), bm3-1 mutant (bm3 cDNA), and the LTR sequence of the B5 element (wXB5 LTR). Nucleotide 520 from which bm3 CDNA strongly diverges from MC1 COMT CDNA is marked with a thin vertical arrow. The position of the COMT gene intron in the corresponding genomic clone MG18 is indicated with a solid arrowhead. The "end" at position 1360 indicates the size of the wild-type COMT cDNA. A 6-bp sequence that could represent a putative polyadenylation signal in bm3 cDNA is underlined. The stop codon in the bm3 COMT sequence is marked with an asterisk. Protein sequence alignment between the wild-type COMT (OMT prot) and $b m 3$-derived chimeric protein (bm3 prot) is shown below the nucleotide sequences. The two double-underlined sequences in wxB5 LTR represent the two 6-bp inverted repeat sequences of the LTR of the B5 element. Vertical double dots indicate a sequence identity. The GenBank, EMBL, and DDBJ accession number of the bm3-1 COMT cDNA is X80669.

indicating that the COMT gene was not affected by these mutations. In the bm3 mutant, however, the length of the band hybridizing to the XS1 probe was clearly shorter and the intensity of the signal greatly reduced (Figure $1 \mathrm{~B}$ ). This analysis indicated that $\sim 600$ nucleotides of the COMT mRNA were lacking in this mutant. An identical result was obtained when the same experiment was carried out with RNA extracted from $b m 3$ mesocotyls or leaves (data not shown). These results suggested that transcription of the COMT gene terminated early or that a post-transcriptional regulation event removed a portion of the COMT mRNA.

To determine which part of the COMT gene was lacking in the COMT mRNA, the same blot was hybridized with a probe corresponding to the $3^{\prime}$ coding region of the COMT gene (SS1, Figure $1 \mathrm{~A}$ ). With such a probe, we observed a similar hybridization pattern for the wild type and $b m 1$ and $b m 2$ mutants (Figure 1B). For the bm3 mutant, a different result was obtained. In this plant, the smaller band previously revealed with the XS1 probe could not be detected, suggesting that at least the SS1-corresponding region in the COMT gene was lacking in the COMT mRNA (Figure 1B).

All of these analyses were also performed with $b m 3$ homozygote mutants maintained in pure W64A and W401 maize lines (bm3-2, Figure $1 C)$. In bm3 roots of both W64A and W401 lines, no mRNA corresponding to COMT was detected with either the XS1 (Figure 1C) or SS1 (data not shown) probe. The same results were observed when RNA analysis was carried out on other tissues (data not shown). All of these results suggested that at least two events affecting the COMT locus may have occurred in bm3 mutants.

\section{A bm3 Mutation Produces a Chimeric COMT mRNA}

To characterize further the bm3 mutation, we cloned and sequenced the bm3 cDNA corresponding to the smaller COMT mRNA detected in the $b m 3$ mutant maintained in the W23 $\times$ L317 hybrid (so-called bm3-1 mutant). A part of the bm3-1 COMT CDNA nucleotide sequence, shown in Figure 2, appears identical to the wild-type COMT CDNA (MC1 CDNA; Collazo et al., 1992), up to nucleotide 519 (Figure 2). Beyond this point, the bm3-1 cDNA nucleotide sequence strongly diverges from the wild-type COMT CDNA and is identical to the long terminal repeat (LTR) of the B5 element, a retrotransposon originally described as interrupting the waxy gene in maize (Varagona et al., 1992). The sequence of the B5 LTR in the COMT mRNA provides a new stop codon and a putative polyadenylation site producing a shorter mRNA, which is consistent with data previously obtained by RNA gel blot analysis. As a consequence, the presence of the B5 LTR drastically changes the open reading frame of the chimeric transcript (Figure 2). The deduced chimeric protein appears shorter than the COMT 
protein (209 amino acids rather than 364) and lacks some of the conserved domains described for all of these proteins (Collazo et al., 1992).

To check for the presence of the LTR of the B5 element in COMT mRNA from the $b m 3-1$ mutant, the region of $b m 3-1 \mathrm{cDNA}$ corresponding to the LTR was used as a probe to hybridize to the previous RNA gel blot. Figure $1 \mathrm{~B}$ shows that an mRNA was detected in bm3-1 roots (Figure 1B) that corresponded to the one previously detected with the XS1 probe (Figure 1B). No COMT mRNA was detected with such a probe in either the wild type or plants carrying other mutations. We also checked for the presence of this transcript in bm3-1 mesocotyls and leaves and obtained an identical result (data not shown). These experiments confirmed the presence of the LTR-corresponding portion of the B5 element in bm3-1 COMT mRNA.

\section{Two bm3 Mutations Arise from Retrotransposition and Deletion in the COMT Gene}

The data obtained by RNA gel blot analysis and bm3-1 cDNA sequencing indicated that COMT mRNA was modified in the bm3-1 mutant maintained in the W23 $\times$ L317 hybrid or was undetectable in the $b m 3-2$ mutant maintained in pure lines. Thus, it was of interest to correlate these data with changes occurring in $b m 3$ alleles by DNA gel blot analysis. Probes were chosen to different regions of the COMT gene and used to compare the structure of both the W23 $\times$ L317 hybrid (wild type and bm3-1 mutant) and W401 (wild type and bm3-2 mutant) alleles. The probes from the coding region were the same as those used for RNA gel blot analysis (XS1 and SS1). An INT probe corresponding to the intron (Figure $1 \mathrm{~A}$ ) present in the COMT gene (Collazo et al., 1992) was also used.

Figure 3 demonstrates that $b m 3$ alleles are polymorphic in the COMT locus because different hybridizing bands were observed, depending on the probe used. In the wild type of the W23 $\times$ L317 hybrid, the same band $(15 \mathrm{~kb})$ was detected using the three probes (Figure 3). In the corresponding bm3-1 mutant, both XS1 and INT probes detected a polymorphic $5.5-\mathrm{kb}$ fragment, whereas SS1 recognized a shorter $3.5-\mathrm{kb}$ fragment. This result indicated that a change occurred in bm3-1 that produced a restriction fragment length polymorphism (RFLP). It also suggested that this modifying event was produced at the junction of the $3^{\prime}$ coding region of the intron, which is consistent with data obtained by $b m 3-1$ cDNA sequencing. The result obtained for bm3-1 could be related to B5 element insertion. Using the region of the bm3-1 cDNA corresponding to the LTR as a control probe, a 5.5-kb hybridizing band was detected. It corresponded to the $5.5-\mathrm{kb}$ hybridizing band detected with both XS1 and INT probes (data not shown).

The same experiment was carried out with the bm3-2 mutant and the corresponding W401 alleles from which no COMT transcript was found (Figure 1C). In the W401 wild-type allele, the three COMT-specific probes detected the same $12-\mathrm{kb}$ fragment (Figure 3 ). In the bm $3-2$ mutant allele, the hybridization pattern obtained with both XS1 and INT probes also indicated a polymorphism because a 13-kb fragment was observed. In bm3-2, however, the $3^{\prime}$ coding region of the COMT gene appeared to be lacking because the 13-kb band that hybridized with the XS1 and INT probes could not be detected. This result suggested that another mutational event, different from the one detected in bm3-1, had occurred in bm3-2. This second change also affects the COMT gene at the junction of the $3^{\prime}$ coding region of the intron. The lack of a hybridizing band when using the SS1 probe indicated that this event might be a deletion of a part of the COMT locus.

The relationship between COMT and $b m 3$ was strengthened by data obtained by mapping the COMT gene on the Pioneer $\mathrm{Hi}-B r e d$ (Johnston, IA) RFLP map with the MC1 cDNA probe (Collazo et al., 1992). Two populations of maize were analyzed (M.L. Katt, Pioneer Hi-Bred Int., personal communication). In the first population, COMT was placed 8.5 map units proximal to BNL05.46 and 2.1 map units distal to BNL15.45. In the second population, it was located 21.4 map units proximal to BNL05.46 and 8.3 map units distal to umc47. All of these RFLP markers are localized in the same region of maize chromosome 4 in which the bm 3 locus has been shown to be mapped (Neuffer et al., 1968; Cherney et al., 1991).
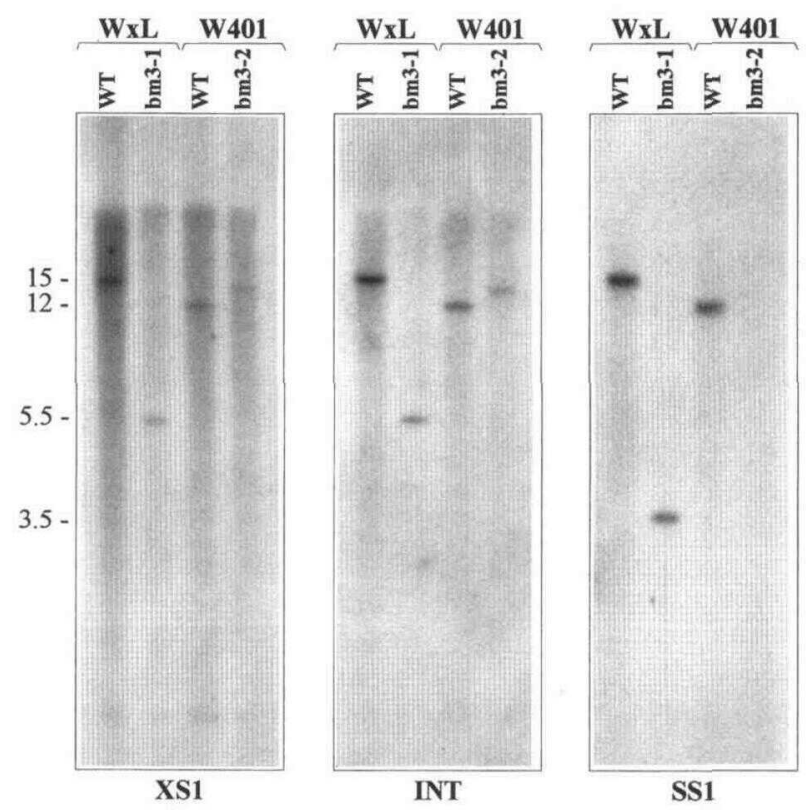

Figure 3. Analysis of Wild-Type and bm3 Maize Genomes by DNA Gel Blot.

Gel blot analysis was performed with $10 \mu \mathrm{g}$ of genomic HindIII-digested DNA from wild-type (WT) and the corresponding bm3 mutants (bm3-1, bm3-2) of the W23 $\times$ L317 hybrid (WxL) and pure W401 (W401) maize lines. The length of the hybridizing bands is indicated at left in kilobases. 


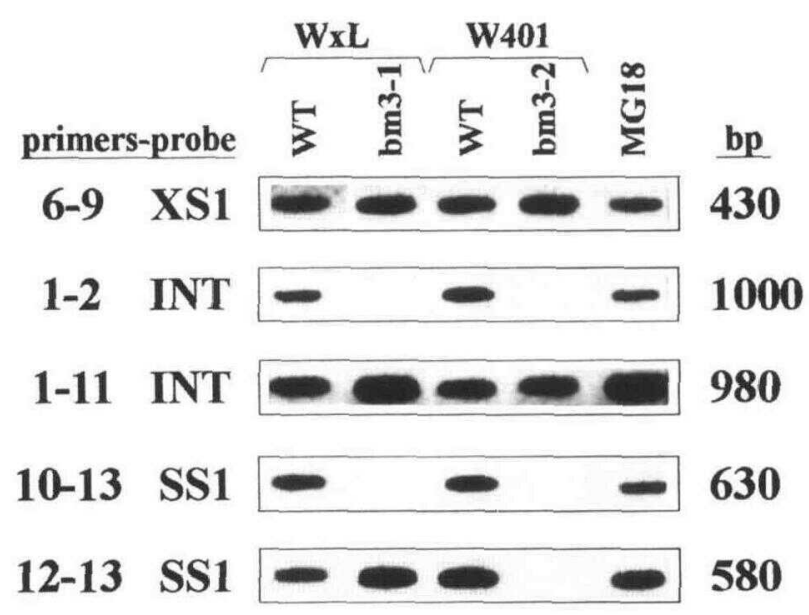

Figure 4. Analysis of Wild-Type and bm3 Mutant Genomes by PCR.

Different regions of the COMT gene were PCR amplified from wild type (WT) and bm3 mutant (bm3-1, bm3-2) genomic DNA isolated from W23 $\times$ L317 hybrid (WXL) and pure W401 (W401) lines. The primers used are described in Figure 1A. Amplifications using the same primers were also performed on genomic clone MG18 (Collazo et al., 1992) as positive controls. PCR products were separated on $1 \%$ agarose gels, blotted onto nylon membranes, and hybridized with the probe corresponding to the amplified region of the COMT gene (see Figure 1A). Probes and primers are indicated at left, and the length of the bands is given at right in base pairs.

\section{Analysis of bm3 Alleles by Polymerase Chain Reaction Amplification}

The data obtained by DNA gel blot analysis reinforced the presumption that two mutational changes affecting COMT occurred in the same region of the gene. To confirm and to characterize further the types of mutations, we used a polymerase chain reaction (PCR) approach involving Hindlll-digested DNA from all lines and various COMT gene-specific primers.

Using two primers surrounding the COMT gene $5^{\prime}$ coding region (primers 6 and 9, Figure 1A), PCR amplification occurred in all alleles (both wild types and mutants), as shown in Figure 4. Two other primers (primers 1 and 2, Figure 1A) surrounding the COMT gene intron were then assayed in all lines. Primer 1 corresponded to the inverse sequence of primer 6 , and primer 2 was chosen from the site of retrotransposon insertion according to the sequence of bm3-1 COMT cDNA. With this primer set, amplification occurred only in the wild-type alleles (Figure 4). No PCR bands were detected in either bm3-1 or bm3-2 mutant alleles, indicating that the region corresponding to primer 2 was modified in both $b m 3$ mutant alleles. When primer 11 (Figure 1A), chosen 12 bp upstream of primer 2, was used along with primer 1 , an amplification product of the expected size was derived from all of the alleles. This result indicated that both bm3-1 and bm3-2 mutations took place at the same region of the COMT gene, within the sequence corresponding to primer 2 downstream of the intron, which is consistent with the DNA gel blot analysis and the sequence of the bm3-1 cDNA.

To confirm the insertion of the retrotransposon in the bm3-1 mutant, amplification of the $3^{\prime}$ coding region was assayed by using primer 10 (complementary to primer 11) and primer 13 , chosen 16 bp upstream of the stop codon of the COMT gene (Figure 1A). Using such primers, the expected product was obtained only from the wild-type alleles (Figure 4). Because the $3^{\prime}$ coding region of the COMT gene could be detected in the bm3-1 mutant by DNA gel blot analysis (Figure 3), we predicted that the absence of the PCR product from the bm3-1 allele was the result of the presence of the B5 retrotransposon. This element, which was described as a 6.1-kb DNA fragment (Varagona et al., 1992), might not be amplifiable under our PCR conditions. Alternatively, a HindIII restriction site introduced due to this insertion may have contributed to the lack of amplification between primer 10 and primer 13 and may explain the RFLP detected between bm3-1 and wild-type alleles.

To characterize further the bm3-2 mutation, bm3-2 and corresponding W401 wild-type alleles were first analyzed using a set of primers (primers 10 and 13, Figure 1A) surrounding the COMT $3^{\prime}$ coding region. Figure 4 indicates that these primers failed to amplify the expected region. This result reinforced the assumption that the $b m 3-2$ mutation was produced in the $3^{\prime}$ coding region of the COMT gene. However, an identical result was obtained from the bm3-1 allele when using these oligonucleotides (Figure 4). Therefore, we tested another set of primers (primers 12 and 13 , Figure $1 A$ ) to understand the difference between the two mutant alleles observed by DNA gel blot analysis (Figure 3). Primer 12 was designed from the $3^{\prime}$ coding region of the COMT gene, downstream of the site of retrotransposon insertion according to the sequence of the bm3-1 COMT cDNA. Amplification between primer 12 and primer 13 normally produced the expected band from both the wild-type and bm3-1 alleles, but the band did not occur from the bm3-2 allele (Figure 4). The lack of a PCR product from the bm3-2 allele indicated that the region containing primers 12 and 13 had been deleted in the corresponding mutant. This is consistent with the absence of a hybridizing band in the DNA gel blot analysis from the bm3-2 allele using the SS1 probe (Figure 3).

\section{The Leaf Midrib is a Location of High COMT Gene Expression}

The aforementioned data indicated that COMT is the gene affected by mutations in bm 3 maize lines. On the other hand, the typical reddish brown pigmentation in bm 3 mutants is associated with the lignified vascular bundles of the leaf midrib (Cherney et al., 1991). Thus, we investigated the cellular distribution of COMT mRNA to check whether it is present in the same cells in which the brown midrib phenotype is visible. For 

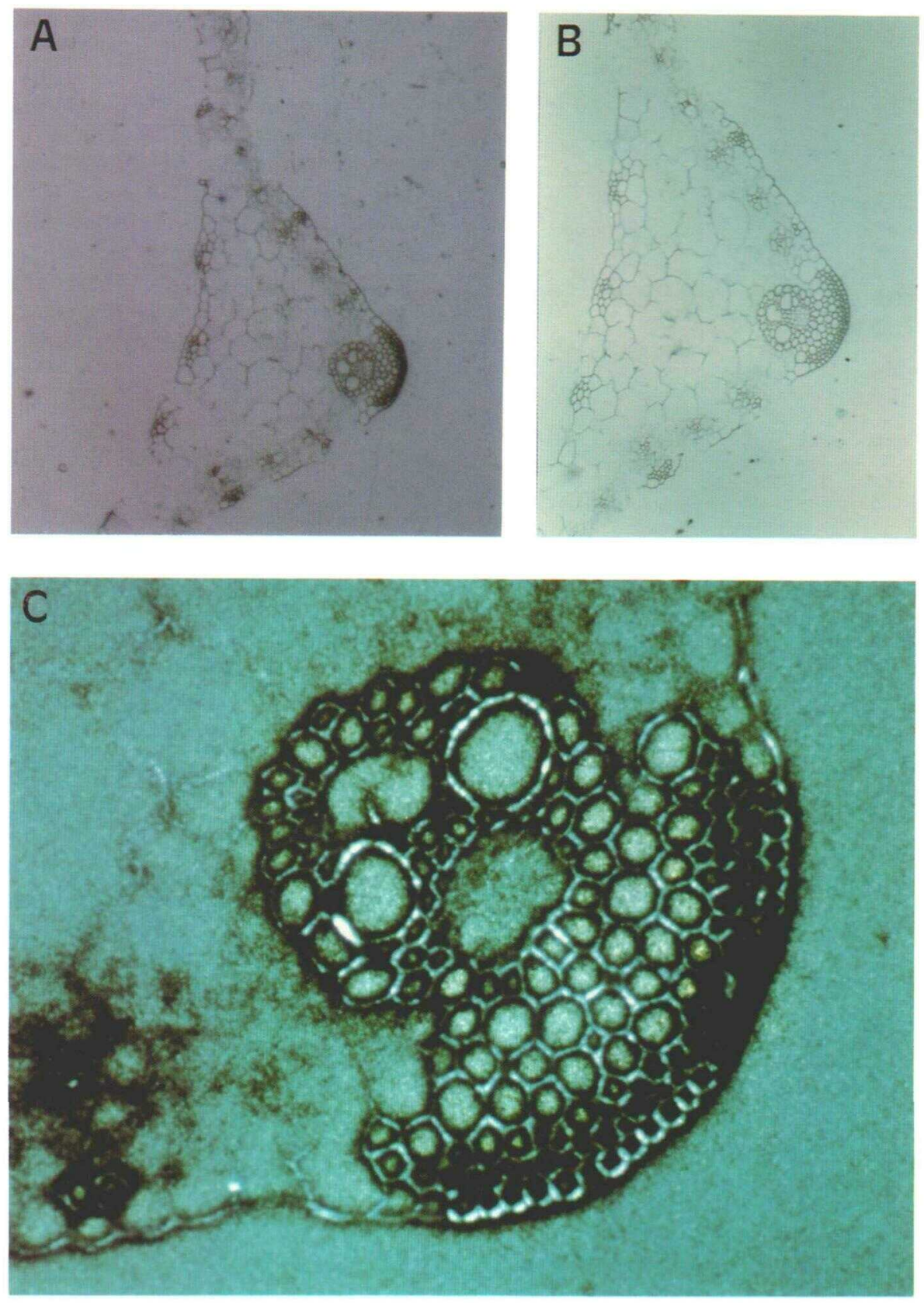

Figure 5. Cellular Expression of the COMT Gene in a Wild-Type Maize Leaf.

Bright-field micrographs of 7- to 8- $\mu$ m-thick tissue sections are shown. In (A) and (C), sections were hybridized with ${ }^{35}$ S-UTP labeled MC1 antisense probes, and in (B), the MC1 sense probe was used. The presence of hybridizing transcripts is indicated by the silver grains that appear as black spots.

(A) Transverse section of a young leaf emerging from the coleoptile.

(B) Same view as shown in (A) but obtained after hybridization with the sense probe as negative control.

(C) Detail of the leaf midrib showing the vascular bundles in which COMT gene expression occurs. 
this purpose, in situ hybridization was carried out with wildtype maize leaves in which COMT mRNA is normally detected by RNA gel blot analysis (Collazo et al., 1992). We used the coding region of MC1 CDNA as a probe (Collazo et al., 1992). Figure 5A demonstrates that COMT mRNA accumulated to high levels in the leaf midrib. The cellular distribution of the COMT mRNA in the leat midrib corresponded to the cells undergoing lignification located around the differentiated xylem vessels. The COMT mRNA was specially abundant in the cells of the sclerenchyma localized under the epidermal layer (Figure 5C).

This result is consistent with the cellular localization of the brown midrib phenotype observed in maize (Cherney et al., 1991). In the leaves of bm3 mutants, subepidermal cells accumulating chimeric COMT mRNA produce a reddish brown pigmentation visible from the surface. In stem or in root, the cells expressing COMT mRNA are internal (data not shown), which explains why the typical reddish brown pigmentation is not visible.

\section{DISCUSSION}

Here, we report the molecular characterization of bm3 mutations in maize and demonstrate that they were produced in the gene coding for the COMT, a key enzyme in the lignin biosynthetic pathway. Two mutant alleles of $b m 3$ were found, designated bm3-1 (reference allele) and bm3-2. In the case of the bm3-1 mutation, a retrotransposon homologous to the B5 family of maize was found to be inserted in the COMT gene. This B5 element previously had been described as interrupting one of the waxy gene introns (Varagona et al., 1992). The insertion of the B5 element in the COMT gene introduced a new stop codon and a polyadenylation signal that produced a shorter COMT mRNA. As a consequence, the COMT protein synthesized in the $b m$ 3-1 mutant is considerably shorter. Specifically, it lacks some of the conserved domains of the OMTs that have been described up to now and that are thought essential for their function (Collazo et al., 1992).

Our work also shows that the second bm 3 mutation (bm3-2) resulted from a deletion that removed at least a portion of the $3^{\prime}$ region of the COMT gene. The results obtained by DNA gel blot and PCR analyses indicated that this bm3-2 mutation was produced in the same region of the COMT gene in which the bm3-1 mutation occurred. As a consequence of both mutations, COMT gene expression was dramatically altered. Using several PCR analyses, we did not observe any change in the COMT gene promoter that might have suggested a modification of gene regulation. However, without further sequence information, it is not possible to rule out this eventuality. Alternatively, the lower level of chimeric COMT transcript detected in the bm3-1 mutant and the absence of COMT mRNA in the bm3-2 mutant in which the COMT gene appeared to be truncated could be the result of post-transcriptional regulation due to the instability of the modified mRNAs.
In both cases, molecular and genetic data demonstrated that bm3 mutations occur in the COMT gene. These data also enabled us to observe the effect of disrupting the COMT gene encoding a key enzyme for lignin biosynthesis and to explain the lower and chemically modified lignin content usually observed in these mutants (Kuc et al., 1968; Gaudillere and Monties, 1989; Barrière et al., 1994). Moreover, these data are consistent with the lower OMT activity previously described in such maize lines (Grand et al., 1985; Lapierre et al., 1988). Thus, the availability of the COMT probe and its identity to $b m 3$ may enable its use in RFLP or PCR-derived methods for breeding programs that include $b m 3$ genes and allow a study of the relationship between the different $b m 3$ alleles.

Aithough OMT activity and lignin content are found to be lower in bm3 mutants, they are still detectable (Grand et al., 1985; Cherney et al., 1991). Considering that lignin-related COMT was shown to be encoded by a single gene in maize (Collazo et al., 1992), we suggest that other methyltransferases exhibiting a lower specificity and efficiency toward COMT substrates may be functional in the lignin biosynthesis pathway. The relative nonspecificity of these enzymes has been demonstrated in plants (Bugos et al., 1991; Edwards and Dixon, 1991; Collazo et al., 1992), and other OMTs normally involved in other pathways, such as flavonoid and isoflavonoid pathways (Vernon and Bohnert, 1992; Held et al., 1993; Maxwell et al., 1993), or disease resistance (Schmitt et al., 1991) could act in an alternative pathway when COMT activity fails. This hypothesis is supported by recent data concerning a caffeoylcoenzymeA 3-0-methyltransferase involved in disease resistance, which has been shown to be effective in the lignification pathway (Ye et al., 1994). The activity of such enzymes might explain the low and modified lignin content typically observed in $b m 3$ mutants.

Antisense technology with genes involved in the lignin pathway has been used in other species and appears effective in lowering the lignin content (Dwivedi et al., 1994; Halpin et al., 1994; Ni et al., 1994). In tobacco, OMT antisense plants were obtained that exhibited a significant reduction in lignin content (Ni et al., 1994). In the same species, CAD antisense plants were produced with a modified lignin structure that probably would be more accessible to chemical degradation (Halpin et al., 1994). For such purposes, antisense methodology appears useful for species in which no mutants related to the lignification process have been described. However, our results indicated that the characterization of lignin-related mutants might be another useful approach that could be designed for species, including maize.

Maize mutants, such as bm3, naturally exhibit the properties of low lignin content and high digestibility (Kuc et al., 1968; Gentinetta et al., 1990; Barrière and Argillier, 1993; Barrière et al., 1994) that are usually the objective of antisense methodology. In some cases, CAD antisense tobacco plants were shown to produce a pigmentation similar to the $b m 3$ mutant in the leaf midrib (Halpin et al., 1994), indicating that comparable events may occur. Because the COMT mRNA appeared highly abundant in the leaf midrib in maize, the characteristic 
pigmentation may be explained as the consequence of the accumulation of an unknown phenolic derivative due to blockage of the normal lignin pathway. Studying well-known mutants related to the lignin biosynthesis pathway to identify mutated genes thus appears an alternative to other methods. Analyzing the effects of disrupting such genes of interest enables possible sites and effects of modification of this important pathway to be evaluated.

\section{METHODS}

\section{Plant Materials}

All of the brown midrib mutants analyzed here are homozygous genotypes. Seed of brown midrib1 (bm1), bm2, and bm3 homozygote mutants maintained in W23 $\times$ L317 hybrids were provided by E.B. Patterson (Maize Genetics Stock Center, University of Illinois, Urbana, IL). The $\mathrm{bm} 3$ allele from the corresponding mutant (the reference allele) is referred to in this study as bm3-1.

Another batch of seed of bm3 homozygote mutants was a gift of Y. Barrière (Institut National de la Recherche Agronomique, INRA, Lusignan, France). The original seeds were furnished to the INRA in 1972 by L.F. Bauman (Department of Agronomy, Purdue University, Lafayette, IN) under code number 95096-102, without further characterization. Later, the $b m 3$ mutants were maintained in W64A and W401 pure inbred maize at the INRA of Lusignan. The $b m 3$ allele from the mutant maintained in the W401 line, which should be given as bm3-95096102 , is referred to in this study as bm3-2, for ease of presentation.

All plants were grown on filter paper for 7 days and stored at $-80^{\circ} \mathrm{C}$

\section{Preparation of OMT Probes}

Probes XS1 and SS1 were obtained by digestion of a caffeic acid O-methyltransferase (COMT) genomic clone MG18 (Collazo et al., 1992) using Xhol/Sacl and Sall restriction enzymes, respectively. Probe INT corresponding to the COMT gene intron was obtained by polymerase chain reaction (PCR) amplification of genomic clone MG18 using primers 1 and 11 (Figure 1A). LTR is the region of the bm3-1 cDNA corresponding to the long terminal repeat of the $B 5$ element. This probe was obtained by PCR amplification of bm3-1 CDNA subcloned in pBluescript SK+ (Stratagene) using primer 1 (Figure 1A) and pBluescript T7 primer. MC1 probe is the full-length COMT CDNA encoding COMT from W64A (Collazo et al., 1992).

\section{RNA Isolation and RNA Blot Analysis}

Total RNA was isolated using lithium chloride precipitation and phenolchloroform extractions according to Verwoerd et al. (1989). Total RNA $(10 \mu \mathrm{g})$ harvested from 7 -day-old roots was separated on a 1.3\% agarose gel following standard procedures (Sambrook et al., 1989) and blotted onto a nylon membrane. The hybridization step $\left(16 \mathrm{hr}\right.$ at $\left.65^{\circ} \mathrm{C}\right)$ was carried out in $250 \mathrm{mM} \mathrm{Na}_{2} \mathrm{HPO}_{4}, 7 \%$ SDS, $1 \mathrm{mM}$ EDTA, $100 \mu \mathrm{g} \mathrm{mL}^{-1}$ salmon sperm DNA containing radiolabeled probe (Feinberg and
Vogelstein, 1984). Washes were performed three times for $20 \mathrm{~min}$ each in $20 \mathrm{mM} \mathrm{Na} 2 \mathrm{HPO}_{4}, 1 \% \mathrm{SDS}, 1 \mathrm{mM}$ EDTA at $65^{\circ} \mathrm{C}$.

cDNA Library Construction, Screening, and DNA Sequencing

Poly(A) ${ }^{+}$RNA was isolated from total RNA using the Poly-A-Tract system (Promega). The cDNA library was constructed in $\lambda$ ZAPII (Stratagene) with $2 \mu \mathrm{g}$ of poly(A) ${ }^{+}$RNA from 7-day-old bm3-1 hybrid roots. The coding XS1 probe was used to screen 300,000 plaques according to standard procedures (Sambrook et al., 1989). Positive clones subcloned in pBluescript $\mathrm{SK}+$ after phagemid in vivo excision were sequenced using the chain termination method (Sambrook et al., 1989) and automatic ALF sequencer (Pharmacia, Sweden). Searches in the data bases (EMBL, GenBank, and DDBJ) and sequence comparisons were performed with the program package, including FASTA 7.2 (Genetics Computer Inc., Madison, WI).

\section{Genomic DNA Isolation and DNA Blot Analysis}

Maize genomic DNA was extracted from 7-day-old leaves, using the standard protocol of Dellaporta et al. (1983). Approximately $10 \mu \mathrm{g}$ of DNA was digested with HindIII and separated on a $0.8 \%$ agarose gel. DNA gel blot hybridization was performed as described for total RNA. Washes were performed at $65^{\circ} \mathrm{C}$ as follows: two washes for $20 \mathrm{~min}$ each in $40 \mathrm{mM} \mathrm{Na} \mathrm{HPO}_{4}, 1 \%$ SDS, 1 mM EDTA, and one wash for $10 \mathrm{~min}$ in $20 \mathrm{mM} \mathrm{Na} \mathrm{HPO}_{4}, 0.5 \%$ SDS, $1 \mathrm{mM}$ EDTA.

\section{Polymerase Chain Reaction Amplifications}

The location of the primers used for PCR amplifications is shown in Figure 1A. PCR analyses were performed with $2 \mu \mathrm{g}$ of Hindlll-digested genomic DNA for more efficient amplifications, and with $50 \mathrm{ng}$ of nondigested DNA for genomic clone MG18 (Collazo et al., 1992). Primers were chosen from the sequence of clone MG18 as follows (from $5^{\prime}$ to $3^{\prime}$ ): primer 1, ATGAACCAGGACAAGGTCCTCATG; primer 2, CATCCCGTACGCCTTGTTGAACGG; primer 6, inverse complementary to primer 1; primer 9, TAATCGTAATAGCCATGGGCTCCA; primer 10, GACGGCGGCATCCCGTTCAAC; primer 11, inverse complementary to primer 10; primer 12, GGCACGGACGCGCGCTTCAAC; primer 13, GCCCAGGCGTTGGCGTAGATG. PCR amplification consisted of 30 cycles at $94^{\circ} \mathrm{C}$ for $2 \mathrm{~min}, 63^{\circ} \mathrm{C}$ for $2 \mathrm{~min}, 72^{\circ} \mathrm{C}$ for $3 \mathrm{~min}$. PCR products were subjected to $1 \%$ agarose gel electrophoresis, transferred onto a nylon membrane, and hybridized with the appropriate probe, as described for total RNA.

\section{In Situ Hybridization}

In situ hybridization was performed on 7- to 8 - $\mu \mathrm{m}$ sections of 7-day-old leaves, using procedures described by Stiefel et al. (1990) and Langdale (1994). Sense and antisense MC1 probes were synthesized with $1 \mu \mathrm{g}$ of linearized DNA using ${ }^{35}$ S-UTP. Labeled transcripts were then hydrolyzed by using an alkali treatment. These transcripts were used in a hybridization step as described by Langdale (1994). Photography was carried out using bright-field microscopy and Ektachrome 160 ASA film (Kodak). 


\section{ACKNOWLEDGMENTS}

We thank Earl B. Patterson and Yves Barrière for providing seeds from maize mutants. We also thank Luis Bosch and Francisco Casañas for their critical reading of the manuscript and Steve Jackson for revising the English. This work was supported by Grant No. Bio94/734 from Plan Nacional de Investigación Científica y Técnica. F.V. is a postdoctoral fellow of the European Community Human Capital and Mobility Program. M.C. was the recipient of a fellowship from the Departament d'Ensenyament of the Generalitat de Catalunya.

Received December 7, 1994; accepted February 24, 1995.

\section{REFERENCES}

Barnes, R.F., Muller, L.D., Bauman, L.F., and Colenbrander, V.F. (1971). In vitro dry matter disappearance of brown-midrib mutants of maize (Zea mays L.). J. Anim. Sci. 33, 881-884.

Barrière, Y., and Argillier, O. (1993). Brown-midrib genes of maize: A review. Agronomie 13, 865-876.

Barrière, Y., Argillier, O., Chabbert, 8., Tollier, M.T., and Monties, B. (1994). Breeding silage maize with brown-midrib genes: Feeding value and biochemical characteristics. Agronomie 14, 11-21.

Bugos, R.C., Chiang, V.L.C., and Campbell, W.H. (1991). cDNA cloning, sequence analysis and seasonal expression of lignin-bispecific caffeic acid/5-hydroxyferulic acid $O$-methyltransferase of aspen. Plant Mol. Biol. 17, 1203-1215

Cherney, J.H., Cherney, D.J.R., Akin, D.E., and Axtell, J.D. (1991). Potential of brown-midrib, low-lignin mutants for improving forage quality. Adv. Agron. 46, 157-198.

Collazo, P., Montoliu, L., Puigdomenèch, P., and Rigau, J. (1992). Structure and expression of the lignin O-methyltransferase gene from Zea mays L. Plant Mol. Biol. 20, 857-867.

Dellaporta, S.L., Wood, J., and Hicks, J.B. (1983). A plant DNA minipreparation. Version II. Plant Mol. Biol. Rep. 1, 19-21.

Dumas, B., Van Doorsselaere, J., Gielen, J., Legrand, M., Fritig, B., Van Montagu, M., and Inzé, D. (1992). Nucleotide sequence of a complementary DNA encoding O-methyltransferase from poplar Plant Physiol. 98, 796-797.

Dwivedi, U.N., Campbell, W.H., Yu, J., Dalta, R.S.S., Bugos, R.C., Chiang, V.L., and Podila, G.K. (1994). Modification of lignin biosynthesis in transgenic Nicotiana through expression of an antisense O-methyltransferase gene from Populus. Plant Mol. Biol. 26, 61-71.

Edwards, R., and Dixon, R.A. (1991). Purification and characterization of S-adenosyl-L-methionine:caffeic acid 3-O-methyltransferase from suspension cultures of alfalfa (Medicago sativa L.). Arch. Biochem. Biophys. 287, 372-379.

Feinberg, A.P., and Vogelstein, B. (1984). A technique for radiolabeling DNA restriction endonuclease fragments to high specific activity. Anal. Biochem. 137, 266-267.

Gaudillere, M., and Monties, B. (1989). Biochemical and biosynthetic studies on lignification of Gramineae. In Plant Cell Wall Polymers: Biogenesis and Biodegradation, Vol. 399, N.G. Lewis and M.G. Paice, eds (Washington, DC: American Chemical Society), pp. 182-192.
Gentinetta, E., Bertolini, M., Rossi, I., Lorenzoni, C., and Motto, M. (1990). Effect of brown midrib-3 mutant on forage quality and yield in maize. J. Genet. Breed. 44, 21-26.

Gowri, G., Bugos, R.C., Campbell, W.H., Maxwell, C.A., and Dixon, R.A. (1991). Stress responses in alfalfa (Medicago sativa L.). X:Molecular cloning and expression of $S$-adenosyl-L-methionine:caffeic acid 3-O-methyltransferase, a key enzyme of lignin biosynthesis. Plant Physiol. 97, 7-14.

Grand, C.P., Parmentier, P., Boudet, A., and Boudet, A.M. (1985) Comparison of lignins and enzymes involved in lignification in normal and brown-midrib (bm3) mutant corn seedlings. Physiol. Veg. 23, 905-911.

Halpin, C., Knight, M.E., Foxon, G.A., Campbell, M.M., Boudet, A.M., Boon, J.J., Chabbert, B., Tollier, M.T., and Schuch, W. (1994). Manipulation of lignin quality by downregulation of cinnamyl alcohol dehydrogenase. Plant J. 6, 339-350.

Held, B.M., Wang, H., John, I., Wurtele, E.S., and Colbert, J.T. (1993). An mRNA putatively coding for an $O$-methyltransferase accumulates preferentially in maize roots and is located predominantly in the region of the endodermis. Plant Physiol. 102, 1001-1008.

Jaeck, E., Dumas, B., Geoffroy, P., and Fritig, B. (1992). Regulation of enzymes involved in lignin biosynthesis: Induction of $O$-methyltransferase mRNAs during the hypersensitive reaction of tobacco to tobacco mosaic virus. Mol. Plant-Microbe Interact. 5, 294-300.

Jorgenson, L.R. (1931). Brown-midrib in maize and its linkage relations. J. Am. Soc. Agron. 23, 549-557.

Kuc, J., and Nelson, O.E. (1964). The abnormal lignins produced by the brown-midrib mutants of maize, I: The brown-midrib 1 mutant. Arch. Biochem. Biophys. 105, 103-113.

Kuc, J., Nelson, O.E., and Flanagan, P. (1968). Degradation of abnormal lignins in the brown-midrib mutants and double mutants of maize. Phytochemistry 7, 1435-1436.

Langdale, J.A. (1994). In situ hybridization. In The Maize Handbook, M. Freeling and V. Walbot, eds (New York: Springer-Verlag), pp. 165-180.

Lapierre, C., Tollier, M., and Monties, B. (1988). Mise en évidence d'un nouveau type d'unité constitutive dans les lignines d'un mutant de mais bm3. C. R. Acad. Sci. 307 (Series III), 723-728.

Lechtenberg, V.L., Muller, L.D., Bauman, L.D., Rhykerd, C.L., and Barnes, R.F. (1972). Laboratory and in vitro evaluation of inbred and F2 populations of brown midrib mutants of Zea mays. Agron. J. 64, 657-660.

Lewis, N.G., and Yamamoto, E. (1990). Lignin: Occurrence, biogenesis and biodegradation. Annu. Rev. Plant Physiol. Plant Mol. Biol. 41, 455-496.

Maxwell, C.A., Harrison, M.J., and Dixon, R.A. (1993). Molecular characterization and expression of alfalfa isoliquiritigenin 2'-O-methyltransferase, an enzyme specifically involved in the biosynthesis of an inducer of Rhizobium meliloti nodulation genes. Plant J. 4, 971-981.

Neuffer, M.G., Jones, L., and Zuber, M.S. (1968). The Mutants of Maize, S. Matthias and H. Hamilton, eds (Madison, WI: Crop Science Society of America)

Ni, W., Paiva, N.L., and Dixon, R.A. (1994). Reduced lignin in transgenic plants containing a caffeic acid $O$-methyltransferase antisense gene. Transgenic Res. 3, 120-126.

Pellegrini, L., Geoffroy, P., Fritig, B., and Legrand, M. (1993). Molecular cloning and expression of a new class of ortho-diphenol- 
O-methyltransferases induced in tobacco (Nicotiana tabacum L.) leaves by infection or elicitor treatment. Plant Physiol. 103, 509-517.

Sambrook, J., Fritsch, E.F., and Maniatis, T. (1989). Molecular Cloning: A Laboratory Manual. (Cold Spring Harbor, NY: Cold Spring Harbor Laboratory).

Sarkanen, K.V., and Ludwig, C.H. (1971). Definition and nomenclature. In Lignins: Occurrence, Formation, Structure and Reactions, K.V. Sarkanen and C.H. Ludwig, eds (New York: Wiley-Interscience), pp. 1-18.

Schmitt, D., Pakusch, A.E., and Matern, U. (1991). Molecular cloning, induction, and taxonomic distribution of caffeoyl-COA 3-O-methyltransierase, an enzyme involved in disease resistance. J. Biol. Chem. 266, 17416-17423.

Stiefel, V., Ruiz-Avila, L., Raz, R., Vallés, M.P., Gómez, J., Pagés, M., Martinez-Izquierdo, J.A., Ludevid, M.D., Langdale, J.A.,
Nelson, T., and Puigdomènech, P. (1990). Expression of a maize cell wall hydroxyproline-rich glycoprotein gene in early leaf and root vascular differentiation. Plant Cell 2, 785-793.

Varagona, M.J., Purugganan, M., and Wessler, S.R. (1992). Alternative splicing induced by insertion of retrotransposons into the maize waxy gene. Plant Cell 4, 811-820.

Vernon, D.M., and Bohnert, H.J. (1992). A novel methyltransferase induced by osmotic stress in the facultative halophyte Mesem. bryanthemum crystallinum. EMBO J. 11, 2077-2085

Verwoerd, T.C., Dekker, B.M.M., and Hokema, A. (1989). A smallscale procedure for the rapid isolation of plant RNAs. Nucl. Acids Res. 17, 2362.

Ye, Z.H, Kneusel, R.E., Matern, U., and Varner, J.E. (1994). An alternative methylation pathway in lignin biosynthesis in Zinnia. Plant Cell 6, 1427-1439. 